\title{
Editorial to the theme section on model-based design of cyber-physical systems
}

\author{
Manfred Broy ${ }^{1} \cdot$ Heinrich Daembkes ${ }^{2}$ Janos Sztipanovits ${ }^{3}$
}

Received: 13 February 2018 / Accepted: 17 February 2018 / Published online: 12 March 2018

(c) Springer-Verlag GmbH Germany, part of Springer Nature 2018

\section{Introduction}

Cyber-physical systems (CPS) are engineered systems where functionalities are emerging from the networked interaction of physical and computational processes. CPS are a pervasive technology advancement, which is impacting today all industrial sectors and almost all aspects of society. While CPS design as a new research area started over a decade ago, the recent emergence of new industrial platforms for creating CPS such as the Internet of things (IoT), industrial Internet (II) and fog computing in the USA and Industrie 4.0 in Europe greatly accelerated the productization and deployment of the technology and created a "gold rush" toward new markets. Since model-based design plays pivotal role in all areas of engineered systems, it is an important issue to examine what are the new challenges for model-based design in CPS?

Model-based design uses formal and sufficiently complete models of physical and computational processes, their environment and their interactions. These models are expected to be mathematically and physically accurate for verifying and testing the behavior of the designed system against established requirements. The main promise of model-based design is a significant decrease or elimination of costly design-build-test-redesign iterations and a higher degree of verified safety, performance and reliability. The ultimate goal is "correct-by-construction", where properties of the synthesized models of the designed system predict the properties of the implemented/manufactured system with sufficient accuracy.

The core CPS challenge for model-based design is that abstraction layer(s) used in the design flow span both cyber and physical aspects. These abstraction layers and the related modeling concepts are selected according to the essential

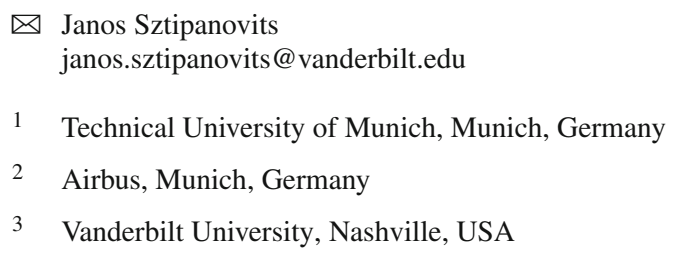

properties that need to be verified and tested during design and monitored during operation. Some of these models (for example, lumped-parameter physical dynamics of controllers of physical processes) represent behaviors that are refined during implementation to software and to physical computation platforms. Similarly, physical interactions may also be virtualized by mapping them to information flows that are connected to the physical world through sensors and actuators. Timing is an essential aspect in many CPS that relies on precisely coordinated interactions between physical and computational processes. In these systems, challenges go well beyond the introduction of physical time abstractions in computing that has a rich history in real-time computing. New challenges and opportunities emerge from integrating the rich concurrency models in computing with time abstractions in physical systems and finding solutions for managing timing uncertainties.

The interaction between the physical and cyber CPS abstraction layers explicitly models the structure and behavior of these physical processes and expresses their relations to cyber models by linking information flows to physical variables via sensors and actuators. However, this is insufficient in system categories where behavior of computing and computation platforms is influenced directly by the environment via physical interactions, such as thermal or electromagnetic effects. Consequently, CPS design flows do not abstract out physicality in computations but consider the implementation side effects of computations and networking on abstracted behaviors including aspects of uncertainty.

\section{In this issue}

This theme section includes four papers addressing fundamental challenges of model-based design due to the heterogeneity of CPS abstraction layers and the complexity of their relationship.

In the paper Basic problems in multi-view modeling, Jan Reineke, Christos Stergion and Stavros Tripakis address 
a frequently used design approach that captures different aspects of the system using a specific modeling view, such as mechanical structure and behavior, software models of embedded controllers or integration models for electrical components. The key challenge they address is consistency across the modeling views. The paper develops a formal framework that enables the precise representation of the semantics of and relation among modeling views. The formalism provides foundation for expressing important concepts in multi-view modeling, such as conformance, view reduction, and orthogonality. The application of the framework is presented in the context of symbolic discrete systems.

Progress toward correct-by-construction model-based design demands the deep integration of abstractions developed in engineering and computing. For example, linking physicality and computation implies using continuous time-space dynamics with discrete, untimed dynamics in the design flow. Simon Bliudze, Sebastien Furic, Joseph Sifakis and Antoine Viel systematically explore the possible approaches in their paper Rigorous design of cyberphysical systems. The paper reviews three main research directions, equation-based modeling exemplified by Modelica, dataflow languages and synchronous languages such as MATLAB/Simulink and hybrid automata modeling integrating event-driven mechanisms and ordinary differential equations (ODE). Authors contrast and evaluate these approaches in three typical steps of CPS design flows: integrated modeling of CPS dynamics, discretization of the models for the purpose of digital implementation and generating executable code for the computational components.

In design flows involving heterogeneous models changing abstraction layers is a common challenge. For example, CPS design may start with physical models using continuous time dynamics that guarantee properties, such as stability. When controller dynamics needs to be implemented computationally, it needs to change to discrete abstractions yielding mathematical models that are approximations of continuous behavior. However, if essential properties, such as stability, can be provably preserved even under the side effects of digital implementation, the required verification and testing effort can be significantly decreased. The paper A model- based design approach for simulation and virtual prototyping of automotive control systems using port-Hamiltonian systems by Siyuan Dai, Zhenkai Zhang and Xenofon Koutsoukos proposes a model-based design methodology based on Port-Hamiltonian Systems that maintains stability as invariant after time discretization and quantization. The paper demonstrates the application of the methodology in automotive control design and validates the results using multimodel simulation.

The complete redesign of abstraction suites and related modeling and simulation tools is not feasible for CPS. Existing tools need to be reused and integrated even if some of their core semantic concepts defer. The paper on Hybrid co-simulation: it's about time by Fabio Cremona Marten Lohstroh, David Brohman, Edward A. Lee, Michael Masin and Stavros Tripakis examines one of the existing simulation integration standard, the Functional Mockup Interface (FMI) from the point of view of simulating systems that mix continuous and discrete behavior. The focus of the paper is modeling of time for hybrid co-simulation with support for discrete and discontinuous signals and instantaneous events. After showing that the current time model in the FMI standard is inadequate for hybrid co-simulation, authors make a concrete proposal for resolving the problems by introducing integer time, automatic choice of time resolution and the use of absent signals.

These papers provide valuable contributions and deeper insights into some of the new challenges of model-based design of CPS. As the contributions show, the impact of heterogeneity of abstractions leads to problems whose solution require rethinking of the theoretical foundations and technology stack dominating the currently separated physical and computation design processes.

Acknowledgements We would like to express our appreciation to the SoSyM editorial office, and, in particular, to Martin Schindler for the excellent support in preparing this theme section. 